\title{
Circadian rhythm and its association with birth and infant outcomes: research protocol of a prospective cohort study
}

\author{
Satvinder Kaur ${ }^{1 *}$, Ai Ni Teoh', Nurul Husna Mohd Shukri², Siti Raihanah Shafie ${ }^{2}$, Normina Ahmad Bustami \\ Masaki Takahashi ${ }^{4}$, Pei Jean $\operatorname{Lim}^{4}$ and Shigenobu Shibata ${ }^{5}$
}

\begin{abstract}
Background: Circadian rhythm plays an important role as our internal body's clock that synchronizes behavior and physiology according to the external 24-h light-dark cycle. Past studies have associated disrupted circadian rhythm with higher risk of miscarriages, preterm birth and low birth weights. This paper described the protocol of a prospective cohort study which aims to determine the circadian rhythm in pregnant women, identify its association with maternal factors during pregnancy, gestational weight gain, birth and infant outcomes.

Methods: Ten government maternal and child health clinics in Kuala Lumpur, Malaysia will be randomly selected. Sample size of 438 first-trimester pregnant women will be followed-up until the birth of their infant. Salivary melatonin and cortisol concentration among subsample will be determined using enzyme-linked immunosorbent assay. Data on sleep quality, psychological distress and morningness/eveningness chronotype of pregnant women will be collected using validated questionnaires. Pedometer will be used to measure 5-day physical activity data. Total gestational weight gain will be determined at the end of pregnancy. Utilization of 3-day food record is to capture meal timing and nutrient intake. All measurements will be done in 2nd and 3rd trimester. Birth outcomes will be collected through clinic records and Centers for Disease Control and Prevention (CDC) Neonatal questionnaire. Infants will be followed-up at 6 and 12 months old to obtain anthropometric measurements.

Discussion: There is a growing recognition of the role of maternal circadian rhythm, which entrains fetal circadian rhythms that may subsequently have long-term health consequences. The present study will identify the effect of circadian rhythm on pregnancy outcomes and infant growth in the first year of life.
\end{abstract}

Keywords: Circadian system, Pregnancy, Chrononutrition, Infant growth, Melatonin, Cortisol

\section{Background}

Circadian rhythm is an endogenous process that has a periodicity of approximately $24 \mathrm{~h}$ [1]. It stimulates anticipation of regular and daily repeating events that take place at approximately the same time of day in organism, allowing the upregulation of most of the major physiological systems in mammals [2]. Circadian system in mammals is organized in a hierarchic manner in which the master (central) clock located in the suprachiasmatic nuclei $(\mathrm{SCN})$ of the anterior hypothalamus govern

\footnotetext{
* Correspondence: satvinderkaur@ucsiuniversity.edu.my

${ }^{1}$ Faculty of Applied Sciences, UCSI University, No. 1, Jalan Menara Gading,

UCSI Heights 56000 Cheras, Kuala Lumpur, Malaysia

Full list of author information is available at the end of the article
}

this process $[1,3]$. The SCN coordinates other central circadian oscillators including the hypothalamus and pituitary gland, which subsequently organizes the peripheral (local) clocks located in the peripheral tissues and aligns the entire circadian system to the external light/dark cycle $[1,3]$. This allows organisms to coordinate endogenous and behavioral activities to the time of day, promoting internal and external synchronization.

Melatonin and cortisol are the most widely used biological phase markers to examine circadian rhythm $[4,5]$. The secretions of these hormones are highly rhythmic, with melatonin exhibiting maximal level in the middle of night and gradual decline towards dawn while cortisol peaks in the second half of the night toward early morning, and

(c) The Author(s). 2020 Open Access This article is distributed under the terms of the Creative Commons Attribution 4.0 International License (http://creativecommons.org/licenses/by/4.0/), which permits unrestricted use, distribution, and reproduction in any medium, provided you give appropriate credit to the original author(s) and the source, provide a link to the Creative Commons license, and indicate if changes were made. The Creative Commons Public Domain Dedication waiver (http://creativecommons.org/publicdomain/zero/1.0/) applies to the data made available in this article, unless otherwise stated. 
declines to half of the peak value in the afternoon $[6,7]$. Both melatonin and cortisol play a role in synchronizing the circadian rhythms in peripheral tissues to the 24-h pattern and likely provide feedback to the SCN [8].

Many emerging studies addressed the recognition of the importance of maternal circadian rhythm on pregnancy and fetal development. Throughout gestation, the fetus is inevitably exposed to maternal rhythms such as body temperature, food intake and melatonin level [9]. Entrained 24-h rhythms of fetal heart rate, movements and hormones were reported in human, non-human primates and sheep, suggesting that maternal circadian signals may contribute to the entrainment of the peripheral clock in fetus [9]. A study by Seron-ferre and his co-researchers proposed that fetal SCN and fetal organs are peripheral maternal circadian oscillators, which are entrained by maternal signals [9]. This results in internal temporal order during fetal growth, which subsequently allows for postnatal integration of the scattered fetal peripheral circadian clocks into an adult-like circadian system [9].

With the growing evidences that demonstrate the long-term impact of pregnancy environment on the development of fetus and the role of circadian system in governing physiological process and metabolic balance, identifying factors associated with circadian rhythm and its relation to pregnancy and infant growth is imperative. Understanding the circadian factors associated with pregnancy has practical importance for preventive care in ensuring a successful pregnancy and optimal development of the fetus, which is essential to lower the risk of developing postnatal diseases of an individual in later life. Therefore, this study intended to fill current research gaps by investigating the association between maternal circadian rhythm with total gestational weight gain, birth and infant outcomes, as well as determining the lifestyle factors affecting maternal circadian rhythm during pregnancy.

\section{Methods/design \\ Study aims}

The aim of the study is to determine the association of stress, physical activity and chrononutrition with circadian rhythm among pregnant women and its impact on total gestational weight gain, birth and infant outcomes. To achieve the aim, the specific research objectives to be investigated in the study are:

1. To determine the circadian rhythm of pregnant women in Kuala Lumpur, Malaysia.

2. To determine the association of stress, physical activity and chrononutrition (energy and nutrient intake, energy and nutrient distribution, meal frequency, eating window, breakfast-skipping, late- night eating and intermittent fasting) with circadian rhythm among pregnant women.

3. To determine the association of irregular circadian rhythm with total gestational weight gain, birth outcomes (birth weight, gestational age at birth and delivery method) and infant outcomes (infant weight, body composition and temperament).

The hypothesis is that mothers with irregular circadian rhythm during pregnancy are associated with higher risk of poor birth outcomes and have unfavorable outcomes, especially on the infant growth and temperament in later life.

\section{Study design}

This prospective cohort study will approach pregnant women from their pregnancy at second and third trimester up to delivery and their infants will be followed up at the age of 6 and 12 months old respectively. The whole study is divided into two phases. Phase I (followup of pregnant women) will be conducted from 2019 to 2020. Data collection will be conducted during the second (gestational week 13 to 27) and third (gestational week 28 to 40) trimester of pregnancy, and upon delivery of the infant. Circadian rhythm of the pregnant women will be assessed using validated questionnaires based on their sleep quality and disturbances, stress, physical activity and chrononutrition. Pre-pregnancy BMI of the pregnant women will be obtained from all participating clinics record. Gestational weight gain of the pregnant women will be monitored throughout the gestation while information on birth outcome will be collected upon delivery. Phase II (follow-up of infant) will be conducted from 2020 to 2021 whereby infants will be followed-up at 6 and 12 months of age to obtain anthropometric measurements. Fig. 1 depicts the flow diagram of the study.

\section{Site and participation selection}

The study will be carried out in the Federal Territory of Kuala Lumpur, which is the capital city of Malaysia located within the central area of Klang Valley. Klang Valley is made up from different ethnic groups, consisting of Malay (50.6\%), Chinese (29.0\%), Indian (11.7\%), other ethnic group (0.7\%) and non-Malaysian citizen (8.0\%) [10]. Kuala Lumpur is the most densely populated city with 7366 people per square kilometer despite a population of 1.79 million people. The population is comprised of $58.5 \%$ of women of reproductive age ranging from 15 to 49 years [11].

Simple random sampling will be used to select maternal and child clinics to reduce sampling bias. The study will be conducted at 10 randomly selected government Maternal and Child Health clinics (Klinik Kesihatan Ibu 


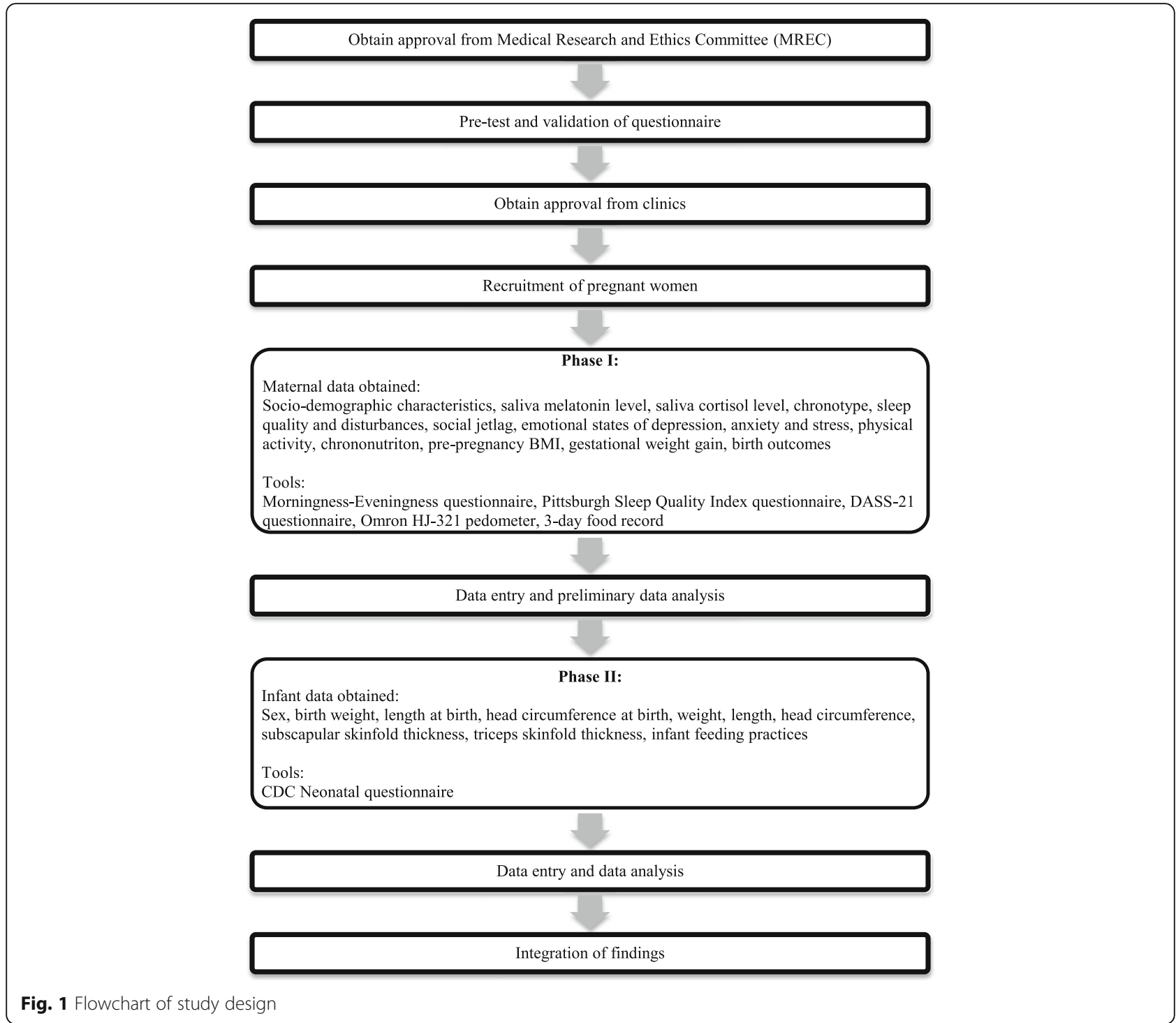

dan Anak) in Kuala Lumpur, Malaysia. Randomization will be stratified by clinic to ensure balanced allocation of participants at each of the ten clinics, with further stratification by ethnicity. The Maternal and Child Health clinics are the primary source providing antenatal and postnatal care to pregnant women. First antenatal checkup will be done in the clinic. Subsequent check-ups and appointments will be made once a month until the 28th week, fortnightly from 29th to 36th week and once a week from the 37th week of pregnancy until delivery. Subjects will be follow-up through clinic's appointment and individual antenatal booklet.

\section{Subject recruitment}

Recruitment of subjects will commence upon obtaining approval from the respective clinics. Purposive sampling method will be used to recruit subjects in their first trimester who fulfil the following inclusion and exclusion criteria, and those who give their consent to participate. Pregnant women who attend the clinics for their routine antenatal care at 1-12 weeks will be approached and invited to participate in this study. The study objectives and procedures will be explained to the subjects and the written consent form will be signed. Information sheet will be provided for their better understanding of the study. Subjects will be asked to sign a written informed consent if they agree to participate in the study on the spot. Additional written consents will be collected from subsample of pregnant women who agree to contribute their saliva samples for circadian melatonin and cortisol rhythm analysis. Sufficient time will be given for subjects to consider their participation in the study and they may contact the research assistant should they agree to participate. Recruitment of subjects began in 
June 2019 and is currently on-going. To minimize loss to follow-up, efforts will be made to maintain personalised contact with the subjects and incentives will be provided.

\section{Inclusion and exclusion criteria}

Eligible subjects must meet all the following inclusion criteria:

1. Malaysian women aged 19-39 years old.

2. Able to read, write and understand English/Malay language.

3. Singleton pregnancy.

4. Nulliparous.

Subjects meeting any of the following criteria will be excluded from participation in the study:

1. Develop any co-morbidities during the study period.

2. Handicap.

3. Use of recreational drugs/cigarette smoking.

4. Having medication that is known to affect sleep and melatonin or cortisol secretion.

5. Shift workers.

6. Transmeridian travel for the prior 3 months.

7. Pre-existing diabetes mellitus, hypertension and anemia prior to pregnancy.

\section{Sample size calculation}

Sample size was calculated using a formula for cohort study with two proportions. Based on the probability of low birth weight among pregnant women with irregular circadian rhythm ( $1=0.0388)$ and probability of low birth weight among pregnant women with regular circadian rhythm ( $\mathrm{p} 2=0.0313$ ), with $95 \%$ power and $5 \%$ significance level, a total of 365 of subjects are required for the study [12]. To compensate non-compliance and non-response among subjects including loss of follow up, the sample size is increased $(+20 \%)$ to 438 pregnant women.

\section{Subsample size calculation}

Given the resource constraints, only a subsample will be studied for the analysis of circadian melatonin and cortisol rhythm from saliva samples. Subsample size is calculated using $G^{*}$ Power software, with expected effect size of 0.82 , type I error rate of 0.05 and a desired power of 0.95 [13]. The total subsample size is 80 as calculated using G*Power software. To compensate for non-compliance and nonresponse among pregnant women, the subsample size is increased (+ 20\%) to 100 .

\section{Study measurements}

Socio-demographic background of subjects, which include their age, ethnicity, educational level, occupation, household income and health history will be obtained through a questionnaire at recruitment. Table 1 shows the details of the variables assessed at each follow-up in this study.

\section{Exposures measures Circadian rhythm}

Circadian rhythm of pregnant women will be assessed using both subjective and objective measurements during second and third trimester of pregnancy. Subjective measurement of chronotype (morning and evening types) will be collected using Morningness-Eveningness questionnaire (MEQ) while objective measurement of circadian rhythm will be determined from salivary melatonin and cortisol rhythm of pregnant women. Chronotype refers to the expression of circadian rhythmicity that may vary among individuals [14]. It was demonstrated in past studies that different chronotypes exhibit vary circadian rhythms of several physiological variables [14]. Besides, sleep parameters are useful as phase markers of the circadian rhythm as well, given that sleep/wake cycle is an important aspect of circadian rhythm [5]. In this study, sleep disturbances and quality of pregnant women during second and third trimester will be determined using Pittsburgh Sleep Quality Index (PSQI) Questionnaire.

Morningness-Eveningness questionnaire The Morningness-Eveningness Questionnaire (MEQ) is the most widely used questionnaire to differentiate individuals with extreme circadian tendencies [15]. This questionnaire is frequently used to correlate with core parameters of human circadian organization such as the timing of sleep and predict the endogenous circadian phase $[5,15]$. The MEQ score is negatively correlated with the objective phase marker in which subjects with a later circadian phase generally scored lower on the MEQ [15]. The MEQ contains 19 questions, most of which elicit preferences in timing of daily activities and sleep. Most questions in MEQ are designed in a preferential manner where respondent is asked to indicate his/her preferred time of rising and bedtime, as well as physical and mental performance and alertness after rinsing and after different activities $[16,17]$. This questionnaire comprises of five behavioral categories: definitive morning (score $=70-86$ ), moderate morning (score $=59$ 69), neither types (score $=42-58$ ), moderate evening $($ score $=31-41)$ and definitive evening $($ score $=16-30)$.

Salivary melatonin and cortisol rhythm In the present study, melatonin and cortisol will be measured using saliva samples. Both melatonin and cortisol can be measured in saliva in their free form [7]. Salivary concentrations have been shown to give an accurate indication of free or biologically active melatonin and cortisol as compared to human serum $[5,18]$. Measuring salivary melatonin and 
Table 1 Summary of data collection and timeline

\begin{tabular}{|c|c|c|c|c|c|c|}
\hline \multirow[b]{2}{*}{ Data } & \multicolumn{3}{|l|}{ Prenatal } & \multicolumn{3}{|l|}{ Postnatal } \\
\hline & Recruitment & 2nd trimester & 3rd trimester & Delivery & 6 months & 12 months \\
\hline \multicolumn{7}{|l|}{ Mothers } \\
\hline Sociodemographic & $\cdot$ & & & & & \\
\hline Date of birth & · & & & & & \\
\hline Ethnicity & $\cdot$ & & & & & \\
\hline Marital status & $\cdot$ & & & & & \\
\hline Educational level & $\cdot$ & & & & & \\
\hline Occupation & $\cdot$ & & & & & \\
\hline Monthly household income & $\cdot$ & & & & & \\
\hline Health history & $\cdot$ & & & & & \\
\hline Pre-pregnancy BMI & $\cdot$ & & & & & \\
\hline Weight at assessment & & $\cdot$ & $\cdot$ & & & \\
\hline DASS-21 & & $\cdot$ & $\cdot$ & & & \\
\hline 3-day food record & & $\cdot$ & · & & & \\
\hline Pittsburgh Sleep Quality Index & & $\cdot$ & $\cdot$ & & & \\
\hline Social jetlag & & $\cdot$ & $\cdot$ & & & \\
\hline Morningness-Eveningness Questionnaire & & $\cdot$ & $\cdot$ & & & \\
\hline Salivary sample & & $\cdot$ & $\cdot$ & & & \\
\hline Step counts & & • & · & & & \\
\hline Neonatal Questionnaire & & & & $\cdot$ & & \\
\hline \multicolumn{7}{|l|}{ Infant } \\
\hline Sex & & & & $\cdot$ & & \\
\hline Mode of delivery & & & & $\cdot$ & & \\
\hline Birth weight & & & & · & & \\
\hline Length at birth & & & & $\cdot$ & & \\
\hline Head circumference at birth & & & & $\cdot$ & & \\
\hline Infant feeding practice & & & & & $\cdot$ & $\cdot$ \\
\hline Weight & & & & & $\cdot$ & $\cdot$ \\
\hline Length/height & & & & & $\cdot$ & $\cdot$ \\
\hline Head circumference & & & & & $\cdot$ & $\cdot$ \\
\hline Waist circumference & & & & & $\cdot$ & $\cdot$ \\
\hline Mid-upper arm circumference & & & & & $\cdot$ & $\cdot$ \\
\hline Subscapular skinfold thickness & & & & & $\cdot$ & $\cdot$ \\
\hline Triceps skinfold thickness & & & & & $\cdot$ & $\cdot$ \\
\hline
\end{tabular}

cortisol can be used to characterize production patterns over each 24-h study period to facilitate comparisons between individual. Moreover, saliva is preferable to serum as a non-invasive alternative to determine melatonin and cortisol level. The taking of blood can cause variation in salivary cortisol level due to the effect of stress $[7,19]$.

Passive drool method will be used whereby subjects need to tilt their head forward and drooled through a funnel into a centrifuge tube [20]. Saliva sample collection will be taken at 2 time points during pregnancy, at second and third trimester respectively. The first sampling process will start at awakening in all cases, followed by 9:00, 15:00, 21:00 and 03: 00 taken at 6 hourly intervals over the 24-h period. This will be done by subjects themselves and each subject will receive individual education regarding the collection process prior to the start of the data collection. To avoid saliva sample contamination with food, subjects are advised to refrain from eating a major meal or brushing their teeth with toothpaste within $30 \mathrm{~min}$ of sample collection [21, 22]. No chocolate or bananas, alcohol, caffeine, nicotine or drinks with artificial colorants should have been taken within the prior $12 \mathrm{~h}$ and on the day of sampling [21-23]. 
During the daytime sampling, saliva samples will be collected under normal light conditions. To minimize light interference with the secretion of salivary hormones, subjects are required to carry out the entire procedure in dim light for collection timed at 03:00 and 21:00 [24]. Subjects will be reminded not to cough up mucus during saliva collection. The centrifuge tube will be covered with aluminium foil and marked with a red line to emphasize the $3 \mathrm{~mL}$ marking. After each collection, the tube containing the saliva sample will be placed in a labelled resealable bag in the family's freezer. Subjects are required to label the tube with the sampling time and date. Subjects will be encouraged to report their actual sampling times, even if there is deviation from the given protocol. Subsequently, the bag with saliva samples will be collected from the subject. The samples will then be centrifuged upon returning to the lab. Supernatant solution will be collected after centrifugation and stored in a $-20{ }^{\circ} \mathrm{C}$ freezer until further analysis. Concentration of salivary melatonin and cortisol will be determined using ELISA kit according to manufacturer-provided standards and protocols. Flowchart illustrating the procedures of passive drool method is shown in Fig. 2.

Pittsburgh sleep quality index questionnaire Pittsburgh sleep quality index (PSQI) is a self-rating questionnaire that measures sleep quality and disturbances over a 1 -month period [25]. PSQI contains 19 items which summarize 7 clinically derived components of sleep quality and patterns: subjective sleep quality, sleep latency, sleep duration, sleep efficiency, sleep disturbances, use of sleep medication and daytime dysfunction. These items are scored from 0 to 3, and then added together to calculate a total PSQI global score ranging from 0 to 21 . It was reported in past studies that the PSQI has similar psychometric properties in both non-pregnant populations and pregnant women [26]. The PSQI has been used on pregnant Asian women in previous studies [26-28]. A validated Malay version of PSQI will be used in the study [29]. The established cutoff of above 5 will be used in this study to depict poor sleep quality in pregnant women. Additionally, bedtime and awake time on workdays and work-free days will be obtained to assess social jetlag (SJL). SJL is calculated as the absolute difference between mid-sleep on work-free days and mid-sleep on workdays [30].

\section{Depression, anxiety, and stress scales (DASS-21) questionnaire}

The Depression, Anxiety and Stress Scale - 21 Items (DASS-21) is a set of three self-report scales designed to

Brush their teeth without toothpaste or rinse mouth with drinking water to remove food residue

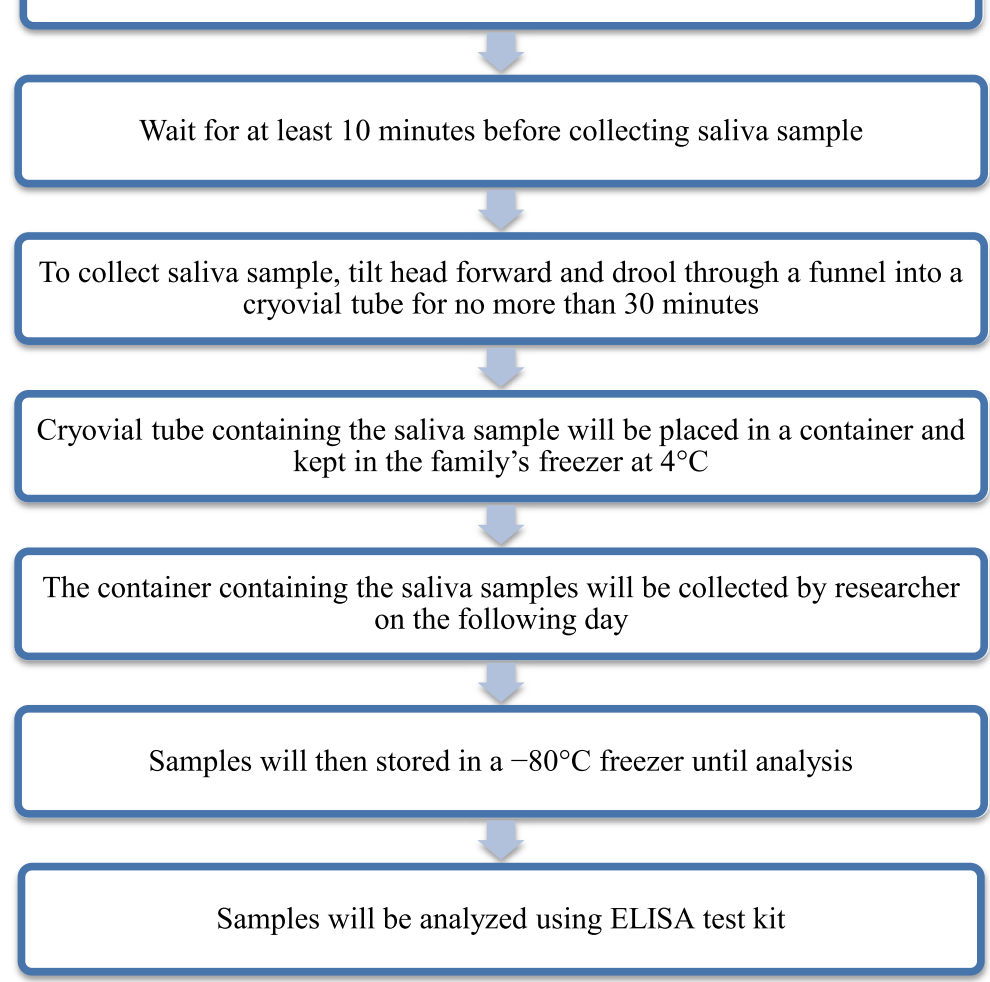

Fig. 2 Flowchart of saliva sample collection by passive drool method 
measure the emotional states of depression, anxiety and stress. DASS-21 contains three subscales that cover depression (7 items), anxiety (7 items), and stress (7 items). Each item is scored from 0 (at all) to 3 (very much). Final score of each subscale will be multiplied by two $(\times 2)$ as DASS-21 is short form version of DASS which has 42 items. The final scores will be classified into different level of severity: normal, mild, moderate, severe and very severe. A valid Malay version of DASS-21 will be used in current study [16]. DASS-21 has been applied in past studies involving pregnant Asian women, including Malaysian pregnant women $[17,31]$.

\section{Measurement of physical activity}

Omron HJ-321 pedometer will be used to measure relative step count in pregnant women. It has been shown to produce accurate measurement of physical activity in the past studies [32-35]. Pedometer is a cost-effective alternative for objective measurement of physical activity and able to capture low-intensity activities including walking [36]. Walking has been reported to be the most popular form of activity for pregnant women in various studies [37-39].

Measurement of relative step count of pregnant women will be taken during second and third trimester. Pedometer readings will be accumulated for a 5 consecutive days during waking hours (excluding water activities), including at least one weekend day, which has previously shown to be sufficient for estimating weekly physical activity [40]. The pedometers will be programmed to an average stride length of $50 \mathrm{~cm}$ as used in the previous study [39]. Subjects will be instructed to carry the pedometer around the neck with a lanyard for 5 days to record activity data. They will be given a simple exercise diary to record their daily step count. Pregnant women are encouraged to maintain their normal daily activities to reduce reactivity bias. A full day was considered as wearing the pedometer for at least eight daytime hours and a half day was considered as $<8 \mathrm{~h}$ but more than $3 \mathrm{~h}$. If worn for $<3 \mathrm{~h}$ a day, this was treated as a missing day. Step count measured by the pedometer will be classified as followed: < 5000/day as "sedentary", 5000-7499 steps/day as "low active", 7500-9999 as "somewhat active" and > 10,000 as physically active based on the criteria proposed by [41].

\section{3-day food record}

A 3-day food record will be used to record timing of meals taken, type of food or beverage and the amount consumed. Dietary intakes will be assessed for 3 nonconsecutive days within a week, comprising two weekdays and one weekend day as the dietary pattern may vary on weekday and weekend day. Subjects will be given instructions on how to record their food intake. Subjects will be encouraged to include detailed descriptions of all foods and beverages including cooking methods, estimated portion size and brand of processed foods and beverages consumed for the past $24 \mathrm{~h}$. Estimation of foods consumed based on household measurement such as cups, plates and tablespoons will be required. To minimize recall bias, subjects will be educated by trained interviewers and they are advised to self-record their diet a the time the food are eaten.

Data that will be identified from the 3-day food record includes eating behaviors including breakfast-skipping, late-night eating and intermittent fasting. Energy and nutrient distribution among the meals will be tabulated. Duration of the last meal of the day to sleeping time will be calculated. Meal frequency as well as eating window will be assessed. Eating window is defined as the time interval between first and last energy-containing meal taken for the day [42].

The estimated amount consumed will then be converted into grams. The mean values for energy and nutrient intake for the three days will be calculated. Malaysian Food Composition Tables [43] and ASEAN Food Composition Tables [44] will be used in dietary analysis. The mean values for energy and nutrient intake will be analyzsed using Nutritionist Pro (First Data Bank Inc., 2011) and then compared with Recommended Nutrient Intake (RNI) for pregnant women [45] to determine intake adequacy. All results will be presented as means, standard deviations and percentages. Ratio between reported total energy intake (EI) and basal metabolic rate (BMR) will be calculated to identify under-reporting of energy intake. BMR is calculated using the BMR equation for Malaysian adults and additional energy requirements during pregnancy will be included in the calculation: $280 \mathrm{kcal} /$ day for second trimester and $470 \mathrm{kcal} /$ day for third trimester [46]. Golderg cut-off point for under-reporting of EI (EI/BMR ratio $<1.2$ ) will be used [47].

\section{Pre-pregnancy body mass index (BMI)}

Pre-pregnancy BMI will be obtained from clinic records. BMI will be computed using Quetelet's Index. World Health Organization (WHO) classification of BMI will be used for weight status classification [48]. BMI classification is divided into six categories based on WHO recommendations as depicted in Table 2.

\section{Outcomes measures}

The main outcomes of this study are birth outcomes and infant outcomes which will be assessed at 6 and 12 months of age, respectively. The secondary outcomes include gestational weight gain (GWG) and infant feeding practices. 
Table 2 BMI classification according to WHO guidelines [47]

\begin{tabular}{ll}
\hline Classification & BMl range $\left(\mathrm{kg} / \mathrm{m}^{2}\right)$ \\
\hline Underweight & $<18.5$ \\
Normal & $18.5-24.9$ \\
Overweight (Pre-obesity) & $25.0-29.9$ \\
Obese class I & $30.0-34.9$ \\
Obese class II & $35.0-39.9$ \\
Obese class III & $\geq 40$ \\
\hline
\end{tabular}

\section{Gestational weight gain}

Range of mean weight gain per week will be determined by deducting weight at assessment with pre-pregnancy maternal weight. Total GWG will be calculated as the differences between maternal weight measured at the last antenatal checkup (at or after 37 weeks period of amenorrhea) and pre-pregnancy weight recorded at the first antenatal appointment at the clinic (measured at < 12 weeks of amenorrhea) [49]. Weight gain recommendation for pregnancy will then be compared with the guidelines established by US Institute of Medicine (IOM). There are three classifications of GEG according to IOM guidelines which are inadequate, adequate and excessive. IOM guidelines on cutoff points for gestational weight gain are illustrated in Table 3.

\section{Birth outcomes}

Birth outcomes such as birth weight, gestational age at birth, delivery method, duration of labor, instrumentassisted labor and other information about the experience during labor will be collected from clinic record. Data on low birth weight, preterm birth and intrauterine growth restriction (IUGR) will be identified through clinic records. Preterm birth is defined as a gestational age less than 37 weeks which may contribute to low birth weight with birth weight less than $2500 \mathrm{~g}$ [50]. IUGR refers to a condition in which the rate of fetal growth is below normal in light of the growth potential of a specific infant as per the race and gender of the fetus [51]. An infant with IUGR is defined as being below the $10 \%$ percentile of the recommended genderspecific birthweight for gestational age according to William's curve $[52,53]$. Focus on the birth outcome will be limited to the 3 major adverse birth outcomes: low birth

Table 3 Institute of Medicine (IOM) guidelines on gestational weight gain [12]

\begin{tabular}{ll}
\hline Pre-pregnancy BMI & $\begin{array}{l}\text { Pregnancy weight } \\
\text { gain goals }(\mathrm{kg})\end{array}$ \\
\hline Underweight & $12.5-18$ \\
Normal & $11.5-16$ \\
Overweight & $7-11.5$ \\
Obese & $5-9$ \\
\hline
\end{tabular}

weight, preterm birth, and intrauterine growth restriction (IUGR). These adverse birth outcomes are the leading causes of perinatal morbidity, mortality, neurodevelopmental impairments and disabilities among newborn babies [53].

\section{CDC neonatal questionnaire}

Neonatal questionnaire from Infant Feeding Practices Study (IFPS) II will be used to assess factors that affect infant feeding choices [54]. Questions on early feeding practices, including breastfeeding status, sources of information and sources of support are included in the questionnaire. These information are crucial as early feeding choices are crucial in influencing infant growth, especially in the first year of life.

\section{Infant outcomes}

Infant anthropometric assessment Data on infant anthropometry will be assessed from infant book record at 6 and 12 months of age respectively. Anthropometry data of infants which comprises of head circumference, length and weight will be collected. Data will then be plot using WHO Growth Charts (include the reference, whereby weight for age, head circumference for age, length for age will be used).

Revised infant behavior questionnaire (IBQ-R) Infant temperament refers to behavioral tendencies that is observed early in life and stable across age [55]. It is an important aspect that might contribute to infant physical growth [55]. Thus, infant temperament will be measured at 14-16 weeks using the validated RIBQ based on a 7point Likert scale, from 1 (never) to 7 (always). Three major dimensions will be assessed: surgency/extraversion, negative affectivity and orienting/regulation. The IBQ-R has been validated for use for infants aged 3 to 12 months old [56] and its reliability has been reported in previous studies [57].

\section{Statistical analysis}

All statistical analysis will be performed using SPSS software version 20 (SPSS Inc., Chicago, IL, USA). Continuous variables are presented as mean \pm SD. Statistical probability level of $p<0.05$ will be considered significant. Normality of continuous data will be tested using Q-Q Plot, histogram and Kolmogorov-Smirnov Test. Data of chronotype, sleep quality, depression, anxiety and stress, physical activity, BMI and GWG will be described in qualitative form. We will perform statistical analysis of the data quantitatively to examine the association between variables.

To determine circadian rhythm, we will perform circadian analysis of salivary melatonin and cortisol levels 
using Acrophase software. Circadian pattern of melatonin and cortisol secretion in 24-h will be represented in a graph. Circadian variables such as baseline (daytime melatonin level), acrophase (clock time at which the melatonin reaches peak level) and amplitude (difference between baseline and peak level) will be extracted from circadian analysis. Diurnal cortisol slope will be tabulated based on two data points (awakening and 21:00) by subtracting value at 21:00 from awakening value, and dividing by the number of hours between these two time points. Chronotype of respondents will be presented as categorical variables of Morning-type (M-type), Neither-type (N-type) and Evening-type (E-type). Student's paired t-test will be used for comparing circadian variables during second and third trimester. Correlation between chronotypes and sleep quality will be assessed using Pearson's correlation test.

We will perform regression to determine the association between circadian variables and depression, anxiety and stress scores and step count. Energy and nutrient intake as well as distribution among meals. Multivariable generalised linear models will be used to examine the associations between chrononutrition data (energy and nutrition intake as well as distribution between meals, duration between the last meal and sleeping time, meal frequency and eating window). We will also perform ANOVA to analyse the association between circadian variables with eating behaviors including breakfastskipping, late-night eating and intermittent fasting.

To further examine the association between the circadian variables with total gestational weight gain, birth outcomes (birth weight, gestational age at birth), infant outcomes (weight, body composition and infant temperament scores), multiple linear regression will be used, with covariates such as maternal age and pre-pregnancy BMI. Odds ratios and 95\% confidence intervals will be used to determine the strength and precision of each association after adjusting for potential confounders.

\section{Discussion}

Pregnancy is a critical period when the system and organs of a new human body develops. Any disruption to the environment in which the fetus grows may result in developmental adaptations that produce permanent alterations to structural and physiological metabolic functions of the fetus [58, 59]. A hypothesis proposed in 1990 by the British epidemiologist David Barker, known as the Barker hypothesis, states that the environment during embryonic and fetal development increases the susceptibility of developing certain postnatal diseases later in life [60]. These effects are referred to as fetal programming which highlights the potential link between maternal factors on fetal growth and subsequently, infant health.
Pregnancy is considered as a period susceptible to chronodisruption as pregnant women often undergo physical, physiological, social and emotional changes, which may alter their usual lifestyle. Sleep problems such as insomnia and sleep deficiency are among the common issues reported during pregnancy due to various factors such as endocrine changes, backache, increase in size, fetal movement, frequent urination, leg cramps, abdominal discomfort, heartburn and vomiting [61, 62]. Sleep/wake cycles are closely intertwined with the circadian system, and disrupted sleep during pregnancy could potentially affect maternal circadian rhythm [5]. Furthermore, due to the lifestyle modernization and common use of artificial lightning, problem of disrupted or irregular circadian rhythm is not limited to shift worker, but may pose a significant health risk on the general public as well.

The strengths of the study include the utilisation of objective and subjective measurement to determine individual aspects of circadian rhythm, namely MorningnessEveningness questionnaire and salivary melatonin and cortisol rhythms. Measurement of melatonin and cortisol levels at specific time points across the day allows the characterization of the full 24-h rhythm, which gives a whole picture of circadian rhythm. The use of pedometer to measure physical activity could minimize under and over-reporting of physical activity which are common when reported using self-assessment questionnaires. Other than using infant weight and body measurements to assess infant outcomes, this study also looks at infant temperament, which is an important aspect that might contribute to infant physical growth. Data on infant feeding practices will be included as early nutrition plays a crucial role in influencing infant growth.

In term of limitations, multiple assessments during second and third trimester of pregnancy could reduce compliance and elevate maternal stress which in turn can result in increased drop-out rates. However, this could be minimized through frequent and positive interactions to encourage continued participation, such as knowledge sharing session with the subjects at the clinic. Another limitations of study is the lack of control for compliance to the protocol of saliva sampling, e.g. by an electric device. However, several steps are undertaken to ensure compliance, including individual education prior to sampling, use of use-friendly sampling kit with easily understood instructions and appointment will be planned to collect the samples from subjects' house, which ease the samples returning process. Besides, this study will include parent report of infant temperament and feeding practices which may cause response bias.

Maternal circadian rhythm and its influence on pregnancy and birth outcomes in human studies are scarce. Majority of the past studies on circadian rhythm focuses on shift work and related abnormalities. Hence, there is 
a need to explore circadian rhythm among the general pregnant women. Data from this study will contribute to the gap in knowledge related to the role of circadian rhythm during pregnancy on birth outcome and infant growth in the first year of life. This study is expected to demonstrate an association between circadian rhythm with total gestational weight gain, birth and infant outcomes. The findings from this study can be used to develop evidence-based recommendations on improving maternal circadian rhythm during pregnancy. By identifying the role circadian rhythm has to play during pregnancy, it could contribute to the development of relevant intervention strategies as well as strengthening existing strategies and policies related to maternal and infant health in Malaysia.

\section{Abbreviations}

ANCOVA: Analysis of covariance; BMI: Body Mass Index; BMR: Basal metabolic rate; CDC: Centre of Disease Control; DASS-21: Depression, Anxiety and Stress Scale - 21 Items; El: Energy intake; ELISA: Enzyme-linked immunosorbent assay; GWG: Gestational weight gain; HKL: General Hospital Kuala Lumpur; IFPS: Infant Feeding Practices Study; IOM: Institute of Medicine; IUGR: Intrauterine growth restriction; MEQ: Morningness-eveningness questionnaire; NPANM: National Plan of Action For Nutrition of Malaysia; PSQI: Pittsburgh sleep quality index; RNI: Recommended nutrient intake; SCN: Suprachiasmatic nuclei; SD: Standard deviation; SJL: Social jetlag; WHO: World Health Organization

\section{Acknowledgements}

We would like to thank the Ministry of Higher Education, Malaysia for supporting this research. The funds were provided through the Fundamental Research Grant Scheme (FRGS/1/2018/SKK06/UCSI/02/2). Abstract of present study was published in Annals of Nutrition and Metabolism as the conference proceedings of Asian Congress of Nutrition 2019.

\section{Authors' contributions}

SK led the conceptualization and design of the study with input from NHS, SRS, NAB, MT, PJL and SS. ANT contributed to the drafting of the manuscript. All authors reviewed and approved the final manuscript.

\section{Funding}

This study is financially supported by the Ministry of Education Malaysia Fundamental Research Grant (FRGS/1/2018/SKK06/UCSI/02/2). There is no involvement of the funder in the conduct of this study. Study protocol has undergone peer review by the funding body.

\section{Availability of data and materials}

Not applicable

\section{Ethics approval and consent to participate}

Approval for the study was obtained from Medical Research and Ethics Committee (KKM/NIHSEC/P19-125), Ministry of Health, Malaysia (protocol number: NMRR-18-3412-45225) on 29th April 2019. Written informed consent will be obtained from the participants prior to data collection. All participants and their background will be kept anonymous and confidential.

\section{Consent for publication}

Not applicable

\section{Competing interests}

The authors declare that they have no competing interests.

\section{Author details}

${ }^{1}$ Faculty of Applied Sciences, UCSI University, No. 1, Jalan Menara Gading, UCSI Heights 56000 Cheras, Kuala Lumpur, Malaysia. ${ }^{2}$ Department of Nutrition and Dietetics, Faculty of Medicine and Health Sciences, Universiti Putra Malaysia, Seri kembangan, Malaysia. ${ }^{3}$ School of Healthy Aging, Medical Aesthetics and Regenerative Medicine, Faculty of Medicine and Health
Sciences, UCSI University, Kuala Lumpur, Malaysia. ${ }^{4}$ Waseda Bioscience Research Institute, Waseda, Singapore. ${ }^{5}$ Department of Electrical Engineering and Biosciences, School of Advanced Engineering and Sciences, Waseda University, Tokyo, Japan.

Received: 26 July 2019 Accepted: 7 February 2020

Published online: 11 February 2020

\section{References}

1. Seron-ferre M, Valenzuela GJ, Torres-Farfan C. Circadian clocks during embryonic and fetal development. Birth Defects Res. 2007;81(3):204-14.

2. Gamble KL, Ryan B, Frank SJ, Young ME. Circadian clock control of endocrine factors. Nat Rev Endocrinol. 2014;10(8):466-75.

3. Tahara Y, Shibata S. Entrainment of the mouse circadian clock: effects of stress, exercise, and nutrition. Free Radic Biol Med. 2018;119:129-38.

4. Klerman EB, Gershengorn HB, Duffy JF, Kronauer RE. Comparisons of the variability of three markers of the human circadian pacemaker. J Biol Rhythm. 2002;17(2):181-93.

5. Hofstra WA, de Weerd AW. How to assess circadian rhythm in humans: a review of literature. Epilepsy Behav. 2008;13(3):438-44.

6. Zisapel N, Tarrasch R, Laudon M. The relationship between melatonin and cortisol rhythms: clinical implications of melatonin therapy. Drug Dev Res. 2005;65(3):119-25.

7. Shimada M, Seki H, Samejima M, Hayase M, Shirai F. Salivary melatonin levels and sleep-wake rhythms in pregnant women with hypertensive and glucose metabolic disorders: a prospective analysis. Biosci Trends. 2016: 2015-01123.

8. Casper RF, Gladanac B. Circadian rhythm and its disruption: impact on reproductive function. Fertil Steril. 2014;102(2):319-20.

9. Seron-ferre M, Mendez N, Abarzua-catalan L, Vilches N, Valenzuela FJ, Reynolds HE, Llanos AJ, Rojas A, Valenzuela GJ, Torres-farfan C. Circadian rhythms in the fetus. Mol Cell Endocrinol. 2012;349(1):68-75.

10. Nor NSM, Ambak R, Aris T. An introduction to the my body is fit and fabulous at home (MyBFF@home): a community-based weight loss intervention study among Malaysian housewives. BMC Womens Health. 2018;18(Supp 1):107.

11. Department of Statistics Malaysia (2010). Population and Housing Census Malaysia 2010 : Distribution and Basic Demographic Characteristic Report. Retrieved from http://pqi.stats.gov.my/result.php?token= cee9490e3181fe0fa1a82c6a3dbf4bfc.

12. Niedhammer I, O'Mahony D, Daly S, Morrison JJ, Kelleher CC. Occupational predictors of pregnancy outcomes in Irish working women in the Lifeways cohort. BJOG Int J Obstet Gynaecol. 2009;116(7):943-52.

13. Simpson W, Frey BN, Steiner M. Mild depressive symptoms during the third trimester of pregnancy are associated with disruptions in daily rhythms but not subjective sleep quality. J Women's Health. 2016;25(6):594-8.

14. Vitale JA, Roveda E, Montaruli A, Galasso L, Weydahl A, Caumo A, Carandente F. Chronotype influences activity circadian rhythm and sleep: differences in sleep quality between weekdays and weekend. Chronobiol Int. 2015;32(3):405-15.

15. Sack RL, Auckley D, Auger RR, Carskadon MA, Wright KP Jr, Vitiello MV, Zhdanova IV. Circadian rhythm sleep disorders: part I, basic principles, shift work and jet lag disorders. Sleep. 2011;30(11):1460-83.

16. Tan PC, Zaidi SN, Azmi N, Omar SZ, Khong SY. Depression, anxiety, stress and hyperemesis gravidarum: temporal and case controlled correlates. PLoS One. 2014;9(3):e92036.

17. Nagandla K, Nalliah S, Loh KY, Majeed ZA, Ismail M, Zubaidah S, Ragavan UD, Krishnan SG. Prevalence and associated risk factors of depression, anxiety and stress in pregnancy. Int J Reprod Contracept Obstet Gynecol. 2016:5(7):2380-8.

18. Grundy A, Tranmer J, Richardson H, Graham CH, Aronson KJ. The influence of light at night exposure on melatonin levels among Canadian rotating shift nurses. Cancer Epidemiol Prev Biomarkers. 2011;20(11):2404-12.

19. Gröschl M. Current status of salivary hormone analysis. Clin Chem. 2008; 54(11):1759-69.

20. Koh DS, Koh GC. The use of salivary biomarkers in occupational and environmental medicine. Occup Environ Med. 2007:64(3):202-10.

21. Zhou JN, Liu RY, van Heerikhuize J, Hofman MA, Swaab DF. Alterations in the circadian rhythm of salivary melatonin begin during middle-age. J Pineal Res. 2003;34(1):11-6. 
22. Mirdamadi K. Salivary melatonin levels in pregnant women with insomnia: a prospective cohort study with two comparison groups (doctoral dissertation). 2016.

23. Bhattarai KR, Kim HR, Chae HJ. Compliance with saliva collection protocol in healthy volunteers: strategies for managing risk and errors. Int J Med Sci. 2018;15(8):823.

24. Skene DJ, Arendt J. Human circadian rhythms: physiological and therapeutic relevance of light and melatonin. Ann Clin Biochem. 2006;43(5):344-53.

25. Buysse D, Reynolds C, Monk T, Berman S, Kupfer D. The Pittsburgh sleep quality index: a new instrument for psychiatric practice and research. Psychiatry Res. 1989;28(2):193-213.

26. Cai S, Tan S, Gluckman PD, Godfrey KM, Saw S, Teoh OH, Chong YS, Meaney MJ, Kramer MS, Gooley JJ. Sleep quality and nocturnal sleep duration in pregnancy and risk of gestational diabetes mellitus. Sleep. 2017;40(2).

27. Panvatvanich S, Lolekha P. Restless legs syndrome in pregnant Thai women: prevalence, predictive factors, and natural course. J Clin Neurol. 2019;15(1): 97-101.

28. Sharma SK, Nehra A, Sinha S, Soneja M, Sunesh K, Sreenivas V, Vedita D. Sleep disorders in pregnancy and their association with pregnancy outcomes: a prospective observational study. Sleep Breath. 2015;20(1):87-93.

29. Yunus RM, Wazid SW, Hairi NN, Choo WY, Hairi FM, Sooryanarayana R, Ahmad SN, Razak IA, Peramalah D, Aziz SA, Mohamad ZL, Mohamad R, Ali ZM, Awang Bulgiba AM. Association between elder abuse and poor sleep: a cross-sectional study among rural older Malaysians. PLoS One. 2017;12(7): e0187782.

30. Komada Y, Okajima I, Kitamura S, Inoue Y. A survey on social jetlag in Japan: a nationwide, cross-sectional internet survey. Sleep Biol Rhythms. 2019:1-6.

31. Nasreen HE, Rahman JA, Rus RM, Kartiwi M, Sutan R, Edhborg M. Prevalence and determinants of antepartum depressive and anxiety symptoms in expectant mothers and fathers: results from a perinatal psychiatric morbidity cohort study in the east and west coasts of Malaysia. BMC Psychiatry. 2018;18(1):195.

32. Widyastuti K, Makhabah DN, Setijadi AR, Sutanto YS, Ambrosino N. Benefits and costs of home pedometer assisted physical activity in patients with COPD. A preliminary randomized controlled trial. Pulmonology. 2018;24(4):211-8.

33. Yilmaz FT, Aydin HT. The effect of a regular walking program on dyspnoea severity and quality of life in normal weight, overweight, and obese patients with chronic obstructive pulmonary disease. Int J Nurs Pract. 2018; 24(3):e12636.

34. Yusoff NAM, Ganeson S, Ismail KF, Juahir H, Shahril MR, Lin LP, Ahmad A Wafa SW, Harith S, Rajikan R. Physical activity level among undergraduate students in Terengganu, Malaysia using pedometer. J Fundam Appl Sci. 2018;10(1S):512-22.

35. Farias-Junior LF, Browne RAV, Freire YA, Oliveira-Dantas FF, Lemos TMAM, Galvão-Coelho NL, Hardcastle SJ, Okano AH, Aoki MS, Costa EC. Psychological responses, muscle damage, inflammation, and delayed onset muscle soreness to high-intensity interval and moderate-intensity continuous exercise in overweight men. Physiol Behav. 2019;199:200-9.

36. Downs DS, Chasan-Taber L, Evenson KR, Leiferman J, Yeo S. Physical activity and pregnancy: past and present evidence and future recommendations. Res Q Exerc Sport. 2012;83(4):485-502.

37. Mottola MF, Campbell MK. Activity patterns during pregnancy. Can J Appl Physiol. 2003:28(4):642-53.

38. Pereira MA, Rifas-shiman SL, Kleinman KP, Rich-edwards JW, Peterson KE, Gillman MW. Predictors of change in physical activity during and after pregnancy: project viva. Am J Prev Med. 2007;32(4):312-9.

39. Harrison CL, Thompson RG, Teede HJ, Lombard CB. Measuring physical activity during pregnancy. J Behav Nutr Phys Act. 2011;8(1):19.

40. Tudor-Locke C, Burkett L, Reis JP, Ainsworth BE, Macera CA, Wilson DK. How many days of pedometer monitoring predict weekly physical activity in adults? Prev Med. 2005;40(3):293-8

41. Tudor-Locke C, Bassett DR. How many steps/day are enough? Sports Med. 2004;34(1):1-8

42. Antoni R, Robertson TM, Robertson MD, Johnston JD. A pilot feasibility study exploring the effects of a moderate time-restricted feeding intervention on energy intake, adiposity and metabolic physiology in freeliving human subjects. J Nutr Sci. 2018;7:1-6.

43. Tee ES, Ismail MN, Nasir MA, Khatijah I. Nutrient composition of Malaysian foods. 4th ed. Kuala Lumpur: Institute for Medical Research; 1997.
44. Puwastien P, Burlingame B, Raroengwichit M, Sungpuag P. ASEAN food composition tables. Thailand: Institute of Nutrition, Mahidol University Thailand; 2000.

45. National Coordinating Committee on Food and Nutrition (NCCFN). Recommended nutrient intakes for Malaysia: Ministry of Health Malaysia; 2017

46. Mohd Ismail N, Ng KK, Chee SS, Roslee R, Zawiah H. Predictive equations for estimation of basal metabolic rate in Malaysian adults. Malays J Nutr. 1998; $4(1$ \& 2):81-90.

47. Goldberg GR, Black AE, Jebb SA, Cole TJ, Murgatroyd PR, Coward WA, Prentice AM. Critical evaluation of data using fundamental principles of energy physiology: 1. Derivation of cut-off limits to identify under-recording Eur J Clin Nutr. 1991:45(12):569-81.

48. World Health Organization. Obesity: preventing and managing the global epidemic: report of a WHO consultation on obesity, Geneva, 3-5 June 1997. Geneva: World Health Organization; 1998.

49. Institute of Medicine (US), National Research Council (NRC): Weight Gain During Pregnancy: Reexamining the Guidelines,. In. Washington, DC: National Academies Press; 2009.

50. Cutland CL, Lackritz EM, Mallett-Moore T, Bardají A, Chandrasekaran R, Lahariya C, Nisar MI, Tapia MD, Pathirana J, Kochhar S, Muñoz FM. Low birth weight: Case definition \& guidelines for data collection, analysis, and presentation of maternal immunization safety data. Vaccine. 2017;35(48Part A):6492.

51. Sharma D, Shastri S, Sharma P. Intrauterine growth restriction: antenatal and postnatal aspects. Clinical Medicine Insights. Pediatrics. 2016;10:CMPedS40070.

52. Williams RL, Creasy RK, Cunningham GC, Hawes WE, Norris FD, Tashiro M. Fetal growth and perinatal viability in California. Obstet Gynecol. 1982;59(5): 624-32.

53. World Health Organization. Physical status: the use and interpretation of anthropometry. Report of a WHO Expert Committee. TRS No. 854. Geneva: World Health Organization; 1995.

54. Centers for Disease Control and Prevention. Infant Feeding Practices Study II: The Questionnaire Atlanta, USA: National Center for Chronic Disease Prevention and Health Promotion. 2009. https://www.cdc.gov/ breastfeeding/data/ifps/questionnaires.htm. Access 5 March 2019.

55. Slining MM, Adair L, Goldman BD, Borja J, Bentley M. Infant temperament contributes to early infant growth: a prospective cohort of African American infants. Int J Behav Nutr Phys Act. 2009;6(1):51.

56. Worobey J, Blajda VM. Temperament ratings at 2 weeks, 2 months, and 1 year: differential stablity of activity and emotionality. Dev Psychol. 1989; 25(2):257.

57. Gartstein MA, Rothbart MK. Studying infant temperament via the revised infant behavior questionnaire. Infant Behav Dev. 2003;26(1):64-86.

58. Kwon EJ, Kim YJ. What is fetal programming?: a lifetime health is under the control of in utero health. Obstet Gynecol Sci. 2017;60(6):506-19.

59. Marciniak A, Patro-Małysza J, Kimber-Trojnar Z, Marciniak B, Oleszczuk J, Leszczynska-Gorzelak B. Fetal programming of the metabolic syndrome. Taiwan J Obstet Gynecol. 2017:56(2):133-8.

60. Almond D, Currie J. Killing me softly: the fetal origins hypothesis. NIH Public Access. 2011;25(3):153-72

61. Pien GW, Schwab RJ. Sleep disorders during pregnancy. Sleep. 2004;27(7): 1405-17.

62. Sloan EP. Sleep disruption during pregnancy. Sleep Med Clin. 2008:3(1):73-80.

\section{Publisher's Note}

Springer Nature remains neutral with regard to jurisdictional claims in published maps and institutional affiliations. 